Bull. Korean Math. Soc. 51 (2014), No. 6, pp. 1669-1687

http://dx.doi.org/10.4134/BKMS.2014.51.6.1669

\title{
ON A NEUMANN PROBLEM AT RESONANCE FOR NONUNIFORMLY SEMILINEAR ELLIPTIC SYSTEMS IN AN UNBOUNDED DOMAIN WITH NONLINEAR BOUNDARY CONDITION
}

\author{
HoAng Quoc ToAn And Bui Quoc Hung
}

\begin{abstract}
We consider a nonuniformly nonlinear elliptic systems with resonance part and nonlinear Neumann boundary condition on an unbounded domain. Our arguments are based on the minimum principle and rely on a generalization of the Landesman-Lazer type condition.
\end{abstract}

\section{Introduction and preliminaries}

Let $\Omega$ be an unbounded domain in $\mathbb{R}^{N}, N \geq 3$ with smooth and bounded boundary $\partial \Omega, \bar{\Omega}=\Omega \cup \partial \Omega$. We consider the existence of weak solutions of Neumann problem for a system of nonuniformly semilinear elliptic equations:

$$
\left\{\begin{array}{l}
-\operatorname{div}\left(h_{1}(x) \nabla u\right)+a_{1}(x) u=\lambda_{11} \theta_{1}(x) u+f(x, u, v)-k_{1}(x) \\
-\operatorname{div}\left(h_{2}(x) \nabla v\right)+a_{2}(x) v=\lambda_{21} \theta_{2}(x) v+g(x, u, v)-k_{2}(x) \quad \text { in } \Omega,
\end{array}\right.
$$

with nonlinear boundary conditions

$$
\left\{\begin{array}{l}
\frac{\partial u}{\partial n}=h_{2}(x) p(x, u, v) \\
\frac{\partial v}{\partial n}=h_{1}(x) q(x, u, v)
\end{array} \quad \text { on } \partial \Omega,\right.
$$

where $\frac{\partial}{\partial n}$ denotes the derivative with respect to the outward unit normal to $\partial \Omega$ and $f, g: \Omega \times \mathbb{R}^{2} \rightarrow \mathbb{R}, p, q: \partial \Omega \times \mathbb{R}^{2} \rightarrow \mathbb{R}$ are Carathéodory functions which will be specified later.

$$
h_{i}(x) \in L_{l o c}^{1}(\bar{\Omega}), h_{i}(x) \geq 1 \text { for a.e } x \in \bar{\Omega}, i=1,2,
$$

$$
a_{i}(x) \in C(\bar{\Omega}), a_{i}(x) \geq a_{0}>0, \forall x \in \bar{\Omega}, a_{i}(x) \rightarrow+\infty \text { as }|x| \rightarrow+\infty, i=1,2,
$$

Received September 25, 2013; Revised April 4, 2014.

2010 Mathematics Subject Classification. 35J20, 35J60, 58E05.

Key words and phrases. semilinear elliptic equation, non-uniform, Landesman-Lazer condition, minimum principle.

Research supported by the National Foundation for Science and Technology Development of Viet Nam (NAFOSTED under grant number 101.01.2011.18). 


$$
\begin{aligned}
& \theta_{i}(x) \in L^{\infty}(\Omega), \theta_{i}(x)>0 \text { for a.e } x \in \bar{\Omega}, i=1,2, \\
& k_{i}(x) \in L^{2}(\Omega), k_{i}(x)>0 \text { for a.e } x \in \bar{\Omega}, i=1,2 .
\end{aligned}
$$

$\lambda_{i 1}$ denotes the first eigenvalue of the problem:

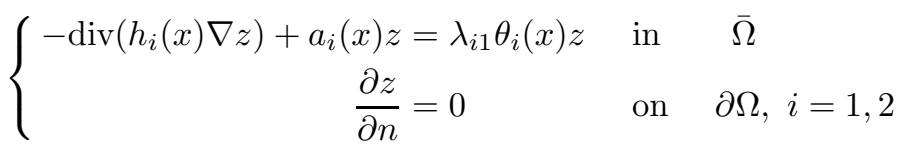

in suitable spaces $E_{i}$ which will be defined below.

We firstly make some comments on the problem (1.1). In the case that $\Omega$ is a bounded domain in $\mathbb{R}^{N}$ and $h(x)=1$ there were extensive studies dealing with the Neumann problem for nonlinear elliptic equation involving the $p$-Laplacian, where different techniques of finding weak solutions are illustrated. When $\Omega$ is unbounded and $h(x) \in L_{l o c}^{1}(\Omega)$, we refer the reader to [6], where the authors have considered Neumann problem for nonuniformly nonlinear equations involving $p$-Laplacian type in an unbounded domain $\Omega \subset \mathbb{R}^{N}$ with smooth and bounded boundary $\partial \Omega$ by using variational techniques via the Mountain Pass Theorem.

On the Landesman-Lazer condition, we refer the reader to $[1,2,3,9,11$, $15,16]$. In $[1,2,3]$ the authors have considered a resonant problem involving $p$-Laplacian in a bounded domain $\Omega \subset \mathbb{R}^{N}$.

$$
\Delta_{p} u=\lambda_{1}|u|^{p-2} u+f(x, u)-h(x),
$$

and the existence of weak solutions $u \in W_{0}^{1, p}(\Omega)$ is shown by taking the wellknown Landesman-Lazer type condition. In $[9,11]$ one has extended some results in $[1,2,3]$ to resonance problems with Dirichlet condition for nonuniformly nonlinear general elliptic equations in divergence form in bounded domain.

The extension to the case of $p$-Laplacian systems on resonance, again with $\Omega$ bounded and Dirichlet boundary condition, was first considered by N. B. Zographopoulos in [16]. Later in [7] D. A. Kandilakis and M. Magiropoulos have studied a quasilinear elliptic system with resonance part and nonlinear boundary condition in an unbounded domain by assuming the nonlinearities $f$ and $g$ depending only one variable $u$ or $v$. In [15] Z.-Q. Ou and C.-L. Tang have considered the same system as in [7] with Dirichlet condition in a bounded domain. In these the existence of weak solutions is obtained by critical point theory under a Landesman-Lazer type condition.

In this paper, by introducing a generalization of Landesman-Lazer type condition, we will prove the existence of weak solutions of Neumann problem for a system on resonance of nonuniformly semilinear elliptic equations in an unbounded domain with general nonlinearities.

Recall that due to $h_{i}(x) \in L_{l o c}^{1}(\Omega)(i=1,2)$ the problem (1.1), (1.2) now is nonuniformly in sense that the Euler-Lagrange functional associated to the problem may be infinity at some $w_{0}=\left(u_{0}, v_{0}\right) \in H^{1}(\Omega) \times H^{1}(\Omega)$. Hence, we must consider problem (1.1), (1.2) in some suitable subspace of $H^{1}(\Omega) \times H^{1}(\Omega)$. 
Denotes by

$$
C_{0}^{\infty}(\bar{\Omega})=\left\{u \in C^{\infty}(\bar{\Omega}): \operatorname{Supp} u \text { compact } \subset \bar{\Omega}\right\},
$$

where $\bar{\Omega}=\Omega \cup \partial \Omega$.

Then $H^{1}(\Omega)$ is a usual Sobolev space which can be defined as the completion of $C_{0}^{\infty}(\bar{\Omega})$ under the norm:

$$
\left.\|u\|=\left(\int_{\Omega}|\nabla u|^{2}+|u|^{2}\right) d x\right)^{\frac{1}{2}} .
$$

We now define following subspaces $E_{i}(i=1,2)$ of $H^{1}(\Omega)$ :

$$
E_{i}=\left\{u \in H^{1}(\Omega): \int_{\Omega}\left[h_{i}(x)|\nabla u|^{2}+a_{i}(x)|u|^{2}\right] d x<+\infty\right\},
$$

where $h_{i}(x)$ and $a_{i}(x)$ satisfy conditions (1.3), (1.4).

By similar arguments as those used in the proof of Proposition 1.2 in [14], we deduce that $E_{i}(i=1,2)$ are Hilbert spaces with the norms:

$$
\|u\|_{E_{i}}=\left(\int_{\Omega}\left[h_{i}(x)|\nabla u|^{2}+a_{i}(x)|u|^{2}\right] d x\right)^{\frac{1}{2}}, u \in E_{i}
$$

and the continuous embeddings $E_{i} \hookrightarrow H^{1}(\Omega) \hookrightarrow L^{q}(\Omega), 2 \leq q \leq 2^{*}(i=1,2)$ hold true.

Moreover the embeddings $E_{i}$ into $L^{2}(\Omega)$ are compact.

Besides since $\partial \Omega$ is bounded and smooth boundary, hence with $R>0$ large enough $\partial \Omega \subset B_{R}(0)$, where $B_{R}(0)$ is ball of radius $R$.

Denote $\Omega_{R}=\bar{\Omega} \cap B_{R}(0)$, the maps $E_{i} \hookrightarrow H^{1}\left(\Omega_{R}\right)$ by $\left.u \rightarrow u\right|_{\Omega_{R}}$ are continuous.

Therefore, from Theorem $A_{8}$ in [12] we deduce that $E_{i} \hookrightarrow L^{2}(\partial \Omega)$ compactly, $i=1,2$.

Remark 1.1. With similar arguments as those used in the proof of Lemma 2.3 in [4], we infer that the functional $J_{i 0}: E_{i} \rightarrow R(i=1,2)$ given by

$$
J_{i 0}(u)=\|\left. u\right|_{E_{i}} ^{2}=\int_{\Omega}\left(h_{i}(x)|\nabla u|^{2}+a_{i}(x)|u|^{2}\right) d x, u \in E_{i}
$$

is weakly lower semicontinuous on $E_{i}$.

Next, we have following proposition which concerns the existence of the first eigenvalue and eigenfunction of the problem (1.7).

Proposition 1.1. Assume that functions $h_{i}(x), a_{i}(x), \theta_{i}(x)(i=1,2)$ satisfy the conditions (1.3), (1.4), (1.5).

Denotes by

$$
\lambda_{i 1}=\inf \left\{\int_{\Omega}\left(h_{i}(x)|\nabla u|^{2}+a_{i}(x)|u|^{2}\right) d x: u \in E_{i}, \int_{\Omega} \theta_{i}(x)|u|^{2} d x=1\right\}, i=1,2 .
$$

Then:

(i) $M_{i}=\left\{u \in E_{i}: \int_{\Omega} \theta_{i}(x)|u|^{2}(x) d x=1\right\} \neq \phi$. 
(ii) There exists $\varphi_{i 1} \in M_{i}, \varphi_{i 1}>0$ in $\bar{\Omega}$ such that:

$$
\int_{\Omega}\left(h_{i}(x)\left|\nabla \varphi_{i 1}\right|^{2}+a_{i}(x)\left|\varphi_{i 1}\right|^{2}\right) d x=\lambda_{i 1} .
$$

Thus $\left(\lambda_{i 1}, \varphi_{i 1}\right)(i=1,2)$ are eigenvalues and eigenfunctions associated with $\lambda_{i 1}$ of the problem (1.7) in $E_{i}$.

Proof. (i) Let $u(x) \in C_{0}^{\infty}(\bar{\Omega}), u \neq 0$. Then $u \in E_{i}$ and $\int_{\Omega} \theta_{i}(x)\left|u^{2}(x)\right| d x>0$.

Choose $\bar{u} \in E_{i}$ as:

$$
\bar{u}(x)=\frac{u(x)}{\left(\int_{\Omega} \theta_{i}(x)\left|u^{2}(x)\right| d x\right)^{\frac{1}{2}}} \quad \text { for } x \in \bar{\Omega} .
$$

Then $\int_{\Omega} \theta_{i}(x)|\bar{u}(x)|^{2} d x=1$. So $\bar{u} \in M_{i}$ and $M_{i} \neq \phi$.

(ii) Let $u_{m} \subset E_{i}$ be a minimizing sequence, i.e.,

$$
\int_{\Omega} \theta_{i}(x)\left|u_{m}(x)\right|^{2} d x=1, m=1,2, \ldots
$$

and $\lim _{m \rightarrow+\infty} \int_{\Omega}\left(h_{i}(x)\left|\nabla u_{m}\right|^{2}+a_{i}(x)\left|u_{m}\right|^{2}\right) d x=\lambda_{i 1}$. So $\left\{u_{m}\right\}$ is bounded in $E_{i}$.

Then, there exists a subsequence $\left\{u_{m_{k}}\right\}_{k}$ such that $\left\{u_{m_{k}}\right\}_{k}$ converges weakly to $\widehat{u}$ in $E_{i}$. Since the embedding $E_{i}$ into $L^{2}(\Omega)$ is compact, the subsequence $\left\{u_{m_{k}}\right\}$ converges strongly to $\widehat{u}$ in $L^{2}(\Omega)$.

Moreover since $\theta_{i}(x) \in L^{\infty}(\Omega)$, we infer that:

$$
1=\lim _{k \rightarrow+\infty} \int_{\Omega} \theta_{i}(x)\left|u_{m_{k}}\right|^{2} d x=\int_{\Omega} \theta_{i}(x)|\widehat{u}|^{2} d x .
$$

So $\widehat{u} \in M_{i}$.

By the minimizing properties and the weakly lower semicontinuity of the functional $J_{i 0}(u)=\int_{\Omega}\left(h_{i}(x)|\nabla u|^{2}+a_{i}(x)|u|^{2}\right) d x$ on $E_{i}$ (see Remark 1.1), we have:

$$
\begin{aligned}
\lambda_{i 1} & =\lim _{k \rightarrow+\infty} \inf \int_{\Omega}\left(h_{i}(x)\left|\nabla u_{m_{k}}\right|^{2}+a_{i}(x)\left|u_{m_{k}}\right|^{2}\right) d x \\
& \geq \int_{\Omega}\left(h_{i}(x)|\nabla \widehat{u}|^{2}+a_{i}(x)|\widehat{u}|^{2}\right) d x \geq \lambda_{i 1} .
\end{aligned}
$$

So we obtain

$$
\lambda_{i 1}=\int_{\Omega}\left(h_{i}(x)|\nabla \widehat{u}|^{2}+a_{i}(x)|\widehat{u}|^{2}\right) d x .
$$

Thus $\widehat{u}$ is a minimizer of (1.8).

Observe further that since $\widehat{u} \in E_{i} \subset H^{1}(\Omega)$ then $|\widehat{u}| \in H^{1}(\Omega)$ (see $[5$, p. 152, Lemma 7.6]). Moreover,

$$
\int_{\Omega}\left(h_{i}(x)|\nabla \widehat{|u|}|^{2}+a_{i}(x) \|\left.\widehat{u}\right|^{2}\right) d x=\int_{\Omega}\left(h_{i}(x)|\nabla \widehat{u}|^{2}+a_{i}(x)|\widehat{u}|^{2}\right) d x<+\infty
$$

and

$$
\int_{\Omega}\left(\theta_{i}(x) \|\left.\widehat{u}\right|^{2}\right) d x=\int_{\Omega}\left(\theta_{i}(x) \widehat{|u|}^{2}\right) d x=1 .
$$


So $|\widehat{u}| \in M_{i}$ is a minimizer too and

$$
\int_{\Omega}\left(h_{i}(x)|\nabla \widehat{|u|}|^{2}+a_{i}(x)|\widehat{|u|}|^{2}\right) d x=\lambda_{i 1}
$$

Applying the Lagrange multiplier rule, we deduce that

$$
\int_{\Omega}\left(h_{i}(x) \nabla|\widehat{u}| \cdot \nabla v+a_{i}(x)|\widehat{u}| \cdot v\right) d x-\lambda_{i 1} \int_{\Omega} \theta_{i}(x)|\widehat{u}| \cdot v d x=0, \forall v \in E_{i} .
$$

This implies that

$$
\left\{\begin{aligned}
-\operatorname{div}\left(h_{i}(x) \nabla|\widehat{u}|\right)+a_{i}(x)|\widehat{u}| & =\lambda_{i 1} \theta_{i}(x)|\widehat{u}| & & \text { in } \Omega, \\
\frac{\partial|\widehat{u}|}{\partial n} & =0 & & \text { on } \partial \Omega .
\end{aligned}\right.
$$

Furthermore, for any $\Omega^{\prime}$ compact $\subset \Omega, h_{i}(x) \in L^{1}\left(\Omega^{\prime}\right), a_{i}(x) \in L^{\infty}\left(\Omega^{\prime}\right),|\widehat{u}| \geq 0$ in $\Omega^{\prime}$ and

$$
-\operatorname{div}\left(h_{i}(x) \nabla|\widehat{u}|\right)+a_{i}(x)|\widehat{u}|=\lambda_{1} \theta_{i}(x)|\widehat{u}|, \text { in } \Omega^{\prime} .
$$

So by the Harnack inequality (see [5, Theorem 8.19 or Theorem 8.20 and Corollary 8.21]), it follows that $|\widehat{u}|>0$ in $\Omega^{\prime}$. This implies that $|\widehat{u}|>0$ in $\bar{\Omega}$.

Denotes $\varphi_{i 1}(x)=|\widehat{u}|$, then $\varphi_{i 1}(x)>0$ in $\Omega$ and $\varphi_{i 1}$ is an eigenfunction of the problem (1.7). The proof of Proposition 1.1 is complete.

On the other hand by similar argument, we also show that the eigenfunctions of $\lambda_{i 1}$ are either positive or negative in $\bar{\Omega}$. Hence, by the compact embedding $E_{i}$ into $L^{2}(\Omega)$ and the standard spectral theory for compact, self-adjoint operators we can infer that for any $i=1,2$ the $\lambda_{i 1}$-eigenfunction $\varphi_{i 1}$ is unique (up to a multiplicitive constant) and

$$
\lambda_{i 1}=\inf _{0 \neq u \in E_{i}} \frac{\int_{\Omega}\left(h_{i}(x)|\nabla u|^{2}+a_{i}(x)|u|^{2}\right) d x}{\int_{\Omega}\left(\theta_{i}(x)|u|^{2}\right) d x}, i=1,2 .
$$

In order to state our main results, let us introduce following some hypotheses on nonlinearities:

$\left(\mathrm{H}_{1}\right)$

(i) $f, g: \bar{\Omega} \times \mathbb{R}^{2} \rightarrow \mathbb{R}, p, q: \partial \Omega \times \mathbb{R}^{2} \rightarrow \mathbb{R}$ are Carathéodory functions:

$$
f(x, 0,0)=0, g(x, 0,0)=0 .
$$

(ii) There exist positive functions $\tau_{1}(x) \in L^{2}(\Omega), \tau_{2}(x) \in L^{2}(\partial \Omega)$ such that:

for all $(s, t) \in \mathbb{R}^{2}$, we have

$|f(x, s, t)| \leq \tau_{1}(x),|g(x, s, t)| \leq \tau_{1}(x)$ for a.e $x \in \Omega$,

$|p(x, s, t)| \leq \tau_{2}(x),|q(x, s, t)| \leq \tau_{2}(x)$ for a.e $x \in \partial \Omega$.

(iii) $f(x, \cdot), g(x, \cdot), p(x, \cdot), q(x, \cdot) \in C^{1}\left(\mathbb{R}^{2}\right)$, and $\forall(s, t) \in \mathbb{R}^{2}$,

$$
\frac{\partial f(x, s, t)}{\partial t}=\frac{\partial g(x, s, t)}{\partial s} \text { for a.e } x \in \Omega,
$$




$$
\frac{\partial p(x, s, t)}{\partial t}=\frac{\partial q(x, s, t)}{\partial s} \text { for a.e } x \in \partial \Omega .
$$

Denotes $\forall(u, v) \in \mathbb{R}^{2}$,

$$
\begin{aligned}
H(x, u, v)= & \frac{1}{2} \int_{0}^{u}[f(x, s, v)+f(x, s, 0)] d s+\frac{1}{2} \int_{0}^{v}[g(x, u, t)+g(x, 0, t)] d t \\
& \text { for a.e } x \in \Omega, \\
R(x, u, v)= & \frac{1}{2} \int_{0}^{u}[p(x, s, v)+p(x, s, 0)] d s+\frac{1}{2} \int_{0}^{v}[q(x, u, t)+q(x, 0, t)] d t \\
& \text { for a.e } x \in \partial \Omega .
\end{aligned}
$$

Remark 1.2. By hypotheses (1.9), (1.10) and (1.11) with some standard computations we deduce that, for all $(u, v) \in \mathbb{R}^{2}$

$$
\begin{aligned}
& \frac{\partial H(x, u, v)}{\partial u}=f(x, u, v), \frac{\partial H(x, u, v)}{\partial v}=g(x, u, v) \text { a.e } x \in \Omega, \\
& \frac{\partial R(x, u, v)}{\partial u}=p(x, u, v), \frac{\partial R(x, u, v)}{\partial v}=q(x, u, v) \text { a.e } x \in \partial \Omega .
\end{aligned}
$$

Now we define, for $i, j=1,2$ :

$$
\begin{gathered}
F_{i j}(x)=\limsup _{\tau \rightarrow+\infty} \frac{1}{\tau} \int_{0}^{\tau}\left[f\left(x,(-1)^{1+i} y \varphi_{11},(-1)^{1+j} \tau \varphi_{21}\right)\right. \\
\left.+f\left(x,(-1)^{1+i} y \varphi_{11}, 0\right)\right] d y, x \in \Omega, \\
G_{i j}(x)=\limsup _{\tau \rightarrow+\infty} \frac{1}{\tau} \int_{0}^{\tau}\left[g\left(x,(-1)^{1+i} \tau \varphi_{11},(-1)^{1+j} y \varphi_{21}\right)\right. \\
\left.+g\left(x, 0,(-1)^{1+i} y \varphi_{21}\right)\right] d y, x \in \Omega, \\
P_{i j}(x)=\limsup _{\tau \rightarrow+\infty} \frac{1}{\tau} \int_{0}^{\tau}\left[p\left(x,(-1)^{1+i} y \varphi_{11},(-1)^{1+j} \tau \varphi_{21}\right)\right. \\
\left.\quad+p\left(x,(-1)^{1+i} y \varphi_{11}, 0\right)\right] d y, x \in \partial \Omega, \\
Q_{i j}(x)=\limsup _{\tau \rightarrow+\infty} \frac{1}{\tau} \int_{0}^{\tau}\left[q\left(x,(-1)^{1+i} \tau \varphi_{11},(-1)^{1+j} y \varphi_{21}\right)\right. \\
F_{i 0}(x)=\limsup _{\tau \rightarrow+\infty} \frac{1}{\tau} \int_{0}^{\tau}\left[f\left(x,(-1)^{1+i} y \varphi_{11}(x), 0\right)\right] d y, x \in \Omega, \\
G_{0 j}(x)=\limsup _{\tau \rightarrow+\infty} \frac{1}{\tau} \int_{0}^{\tau}\left[g\left(x, 0,(-1)^{1+j} y \varphi_{21}(x)\right)\right] d y, x \in \Omega, \\
P_{i 0}(x)=\limsup _{\tau \rightarrow+\infty} \frac{1}{\tau} \int_{0}^{\tau}\left[p\left(x,(-1)^{1+i} y \varphi_{11}(x), 0\right)\right] d y, x \in \partial \Omega, \\
Q_{0 j}(x)=\limsup _{\tau \rightarrow+\infty} \frac{1}{\tau} \int_{0}^{\tau}\left[q\left(x, 0,(-1)^{1+j} y \varphi_{21}(x)\right)\right] d y, x \in \partial \Omega .
\end{gathered}
$$


Set $h(x)=h_{1}(x) h_{2}(x)$ and

$$
\begin{aligned}
L_{11}= & \int_{\Omega}\left(F_{11}(x) \varphi_{11}(x)+G_{11}(x) \varphi_{21}(x)\right) d x \\
& +\int_{\partial \Omega} h(x)\left[P_{11}(x) \varphi_{11}(x)+Q_{11}(x) \varphi_{21}(x)\right] d s, \\
L_{22}= & \int_{\Omega}\left(F_{22}(x) \varphi_{11}(x)+G_{22}(x) \varphi_{21}(x)\right) d x \\
& +\int_{\partial \Omega} h(x)\left[P_{22}(x) \varphi_{11}(x)+Q_{22}(x) \varphi_{21}(x)\right] d s, \\
L_{12}= & \int_{\Omega}\left(F_{12}(x) \varphi_{11}(x)-G_{12}(x) \varphi_{21}(x)\right) d x \\
& +\int_{\partial \Omega} h(x)\left[P_{12}(x) \varphi_{11}(x)-Q_{12}(x) \varphi_{21}(x)\right] d s, \\
L_{21}= & \int_{\Omega}\left(F_{21}(x) \varphi_{11}(x)-G_{21}(x) \varphi_{21}(x)\right) d x \\
& +\int_{\partial \Omega} h(x)\left[P_{21}(x) \varphi_{11}(x)-Q_{21}(x) \varphi_{21}(x)\right] d s,
\end{aligned}
$$

$$
\begin{aligned}
L_{i 0} & =\int_{\Omega} F_{i 0}(x) \varphi_{11}(x) d x+\int_{\partial \Omega} h(x) P_{i 0}(x) \varphi_{11}(x) d s, i=1,2, \\
L_{0 j} & =\int_{\Omega} G_{0 j}(x) \varphi_{21}(x) d x+\int_{\partial \Omega} h(x) Q_{0 j}(x) \varphi_{21}(x) d s, i=1,2 .
\end{aligned}
$$

$\left(\mathrm{H}_{2}\right)$ Assume that the following potential Landesman-Lazer type conditions hold:

(i)

$$
\begin{aligned}
& L_{11}<2 \int_{\Omega}\left[k_{1}(x) \varphi_{11}(x)+k_{2}(x) \varphi_{21}(x)\right] d x<L_{22}, \\
& L_{12}<2 \int_{\Omega}\left[k_{1}(x) \varphi_{11}(x)-k_{2}(x) \varphi_{21}(x)\right] d x<L_{21} .
\end{aligned}
$$

(ii)

$$
\begin{aligned}
& L_{10}<\int_{\Omega} k_{1}(x) \varphi_{11}(x) d x<L_{20}, \\
& L_{01}<\int_{\Omega} k_{2}(x) \varphi_{21}(x) d x<L_{02},
\end{aligned}
$$

where $\varphi_{i 1}$ are eigenfunctions associated with $\lambda_{i 1}$ of the problem (1.7) in $E_{i}$, $i=1,2$. 
Definition 1.1. Function $w=(u, v) \in E=E_{1} \times E_{2}$ is called a weak solution of the problem (1.1), (1.2) if and only if

$$
\begin{aligned}
& \int_{\Omega}\left(h_{1}(x) \nabla u \nabla \varphi(x)+a_{1}(x) u \varphi(x)\right) d x+\int_{\Omega}\left(h_{2}(x) \nabla v \nabla \psi(x)+a_{2}(x) v \psi(x)\right) d x \\
& -\lambda_{11} \int_{\Omega} \theta_{1}(x) u \varphi(x) d x-\lambda_{21} \int_{\Omega} \theta_{2}(x) v \psi(x) d x \\
& -\int_{\Omega}[f(x, u, v) \varphi+g(x, u, v) \psi(x)] d x+\int_{\Omega}\left[k_{1}(x) \varphi(x)+k_{2}(x) \psi(x)\right] d x \\
& -\int_{\partial \Omega} h(x)[p(x, u, v) \varphi(x)+q(x, u, v) \psi(x)] d s=0
\end{aligned}
$$

for all $(\varphi, \psi) \in C_{0}^{\infty}(\bar{\Omega}) \times C_{0}^{\infty}(\bar{\Omega})$.

Remark 1.3. If $\left(u_{0}, v_{0}\right) \in C^{2}(\bar{\Omega}) \times C^{2}(\bar{\Omega})$ satisfies the condition (1.17), hence $\left(u_{0}, v_{0}\right)$ is a classical solution of the problem (1.1), (1.2). (See Remark 1.1 in [6].)

Our main result is given by the following theorem.

Theorem 1.1. Assuming conditions $\left(\mathrm{H}_{1}\right),\left(\mathrm{H}_{2}\right)$ are fulfilled. Then the problem (1.1), (1.2) admits at least a nontrivial weak solution in $E=E_{1} \times E_{2}$.

Proof of Theorem 1.1 is based on variational techniques and the Minimum Principle.

\section{Proof of the main result}

The Euler-Lagrange functional associated to the problem (1.1), (1.2), I : $E \rightarrow R$ is given by

$$
\begin{aligned}
I(w)= & \frac{1}{2} \int_{\Omega}\left(h_{1}(x)|\nabla u|^{2}+a_{1}(x)|u|^{2}\right) d x+\frac{1}{2} \int_{\Omega}\left(h_{2}(x)|\nabla v|^{2}+a_{2}(x)|v|^{2}\right) d x \\
& -\frac{\lambda_{11}}{2} \int_{\Omega} \theta_{1}(x)|u|^{2} d x-\frac{\lambda_{21}}{2} \int_{\Omega} \theta_{2}(x)|v|^{2} d x-\int_{\Omega} H(x, u, v) d x \\
& +\int_{\Omega}\left(k_{1}(x) u+k_{2}(x) v\right) d x-\int_{\partial \Omega} h(x) R(x, u, v) d s, \forall w=(u, v) \in E
\end{aligned}
$$

where $h(x)=h_{1}(x) h_{2}(x), H(x, u, v), R(x, u, v)$ are given by (1.11).

Denotes

$$
\begin{aligned}
J(w)= & \frac{1}{2} \int_{\Omega}\left(h_{1}(x)|\nabla u|^{2}+a_{1}(x)|u|^{2}\right) d x \\
& +\frac{1}{2} \int_{\Omega}\left(h_{2}(x)|\nabla v|^{2}+a_{2}(x)|v|^{2}\right) d x, w=(u, v) \in E .
\end{aligned}
$$




$$
\begin{aligned}
T(w)= & -\frac{\lambda_{11}}{2} \int_{\Omega} \theta_{1}(x)|u|^{2} d x-\frac{\lambda_{21}}{2} \int_{\Omega} \theta_{2}(x)|v|^{2} d x-\int_{\Omega} H(x, u, v) d x \\
& +\int_{\Omega}\left(k_{1}(x) u+k_{2}(x) v\right) d x-\int_{\partial \Omega} h(x) R(x, u, v) d s, w=(u, v) \in E .
\end{aligned}
$$

By hypotheses $\left(\mathrm{H}_{1}\right)$ the functions $J, T$ and then $I=J+T$ are well-defined on $H$.

Remark 2.1. By Remark 1.1, the functional $J(w), w \in E$ given by (2.1) is weak lower semicontinuous on $E$. Moreover from hypothesis $\left(\mathrm{H}_{1}\right)$ with some standard arguments we deduce that the functional $T(w), w \in E$ given by (2.3) is also weak lower semicontinuous on $E$. Thus the functional $I=J+T$ is weak lower semicontinuous on $E$.

The following proposition which concerns the smoothness of the functional $I$ on $E$.

Proposition 2.1. The Euler-Lagrange functional I given by (2.1) is Fréchet differentiable on $E$ and we have:

$$
\begin{aligned}
\left(I^{\prime}(w), \bar{w}\right)= & \int_{\Omega}\left(h_{1}(x) \nabla u \nabla \bar{u}+a_{1}(x) u \bar{u}\right) d x+\int_{\Omega}\left(h_{2}(x) \nabla v \nabla \bar{v}+a_{2}(x) v \bar{v}\right) d x \\
& -\lambda_{11} \int_{\Omega} \theta_{1}(x) u \bar{u} d x-\lambda_{21} \int_{\Omega} \theta_{2}(x) v \bar{v} d x \\
& -\int_{\Omega}[f(x, w) \bar{u}+g(x, w) \bar{v}] d x+\int_{\Omega}\left(k_{1}(x) \bar{u}+k_{2}(x) \bar{v}\right) d x \\
& -\int_{\partial \Omega} h(x)[p(x, w) \bar{u}+q(x, w) \bar{v}] d s, \forall w=(u, v), \bar{w}=(\bar{u}, \bar{v}) \in E .
\end{aligned}
$$

Proof. With similar arguments as those used in the proof of Proposition 2.2(iii) in [14] we deduce that the functional $J$ given by (2.2) is Gateaux differentiable on $E$ and whose the Gateaux derivative is given by:

$$
\begin{aligned}
\left(J^{\prime}(w), \bar{w}\right)= & \int_{\Omega}\left(h_{1}(x) \nabla u \nabla \bar{u}+a_{1}(x) u \bar{u}\right) d x \\
& +\int_{\Omega}\left(h_{2}(x) \nabla v \nabla \bar{v}+a_{2}(x) v \bar{v}\right) d x, w=(u, v), \bar{w}=(\bar{u}, \bar{v}) \in E .
\end{aligned}
$$

Now let $\left\{w_{m}=\left(u_{m}, v_{m}\right)\right\}$ be a sequence converging to $w=(u, v)$ in $E$, i.e.,

$$
\begin{aligned}
\lim _{m \rightarrow+\infty}|| w_{m}-w \|_{E}^{2}= & \lim _{m \rightarrow+\infty}\left\{\int_{\Omega}\left(h_{1}(x)\left|\nabla\left(u_{m}-u\right)\right|^{2}+a_{1}(x)\left|u_{m}-u\right|^{2}\right) d x\right. \\
& \left.+\int_{\Omega}\left(h_{2}(x)\left|\nabla\left(v_{m}-v\right)\right|^{2}+a_{2}(x)\left|v_{m}-v\right|^{2}\right) d x\right\}=0 .
\end{aligned}
$$


Then by some simple computations we have:

$$
\begin{aligned}
\left|\left(J^{\prime}\left(w_{m}\right)-J^{\prime}(w)\right), \bar{w}\right|= & \mid \int_{\Omega}\left(h_{1}(x) \nabla\left(u_{m}-u\right) \nabla \bar{u}+a_{1}(x)\left(u_{m}-u\right) \bar{u}\right) d x \\
& +\int_{\Omega}\left(h_{2}(x) \nabla\left(v_{m}-v\right) \nabla \bar{v}+a_{2}(x)\left(v_{m}-v\right) \bar{v}\right) d x \mid \\
\leq & 4\|\bar{w}\|_{E} \cdot\left\|w_{m}-w\right\|_{E} \text { for all } \bar{w}=(\bar{u}, \bar{v}) \in E .
\end{aligned}
$$

This implies that

$$
\left\|J^{\prime}\left(w_{m}\right)-J^{\prime}(w)\right\|_{E^{*}} \leq C\left\|w_{m}-w\right\|_{E}
$$

Let $m \rightarrow+\infty$ we obtain: $\lim _{m \rightarrow+\infty} J^{\prime}\left(w_{m}\right)=J^{\prime}(w)$ in $E^{*}$.

Hence $J^{\prime}$ is continuous on $E$. Thus $J \in C^{1}(E, R)$.

Besides, from hypotheses $\left(H_{1}\right)$ and $(1.5),(1.6)$ on the functions $f, g, p$, $q, \theta_{1}, \theta_{2}$ and $k_{1}(x), k_{2}(x)$, for some standard computations we infer that the functional $T$ given by (2.3) is Fréchet differentiable on $E$ and we get:

$$
\begin{aligned}
\left(T^{\prime}(w), \bar{w}\right)= & -\lambda_{11} \int_{\Omega} \theta_{1}(x) u \bar{u} d x-\lambda_{21} \int_{\Omega} \theta_{1}(x) v \bar{v} d x \\
& -\int_{\Omega}[f(x, w) \bar{u}+g(x, w) \bar{v}] d x+\int_{\Omega}\left(k_{1}(x) \bar{u}+k_{2}(x) \bar{v}\right) d x \\
& -\int_{\partial \Omega} h(x)[p(x, w) \bar{u}+q(x, w) \bar{v}] d s, \forall w=(u, v), \bar{w}=(\bar{u}, \bar{v}) \in E .
\end{aligned}
$$

Finally, the functional $I=J+T \in C^{1}(E, R)$ and we have (2.4). Proposition 2.1 is proved.

Remark 2.2. By Proposition 2.1 the critical points of the Euler-Lagrange functional $I$ are precisely the weak solutions of the problem $(1.1),(1.2)$.

Proposition 2.2. The functional I given by (2.1) is coercive on E provided that conditions $\left(\mathrm{H}_{1}\right)$ and $\left(\mathrm{H}_{2}\right)$ hold true.

Proof. By contradiction we assume that the functional $I$ is not coercive on $E$. Then there exists a sequence $\left\{w_{m}=\left(u_{m}, v_{m}\right)\right\}_{m=1}^{\infty}$ in $E$ such that

$$
\left\|w_{m}\right\|_{E} \rightarrow+\infty \text { as } m \rightarrow+\infty \text { and } I\left(w_{m}\right) \leq c
$$

where $c$ is positive constant.

Let $\widehat{w}_{m}=\frac{w_{m}}{\left\|w_{m}\right\|_{E}}=\left(\widehat{u}_{m}, \widehat{v}_{m}\right)$, that is $\widehat{u}_{m}=\frac{u_{m}}{\left\|w_{m}\right\|_{E}}, \widehat{v}_{m}=\frac{v_{m}}{\left\|w_{m}\right\|_{E}}, m=$ $1,2, \ldots$ Thus $\left\{\widehat{w}_{m}\right\}_{m}$ is a bounded sequence in $E$.

Then there exists a subsequence $\left\{\widehat{w}_{m_{k}}\right\}_{k}=\left\{\left(\widehat{u}_{m_{k}}, \widehat{v}_{m_{k}}\right)\right\}$ which converge weakly to some $\widehat{w}=(\widehat{u}, \widehat{v})$ in $E=E_{1} \times E_{2}$.

Since the embeddings $E_{i},(i=1,2)$ into $L^{2}(\Omega)$ and $L^{2}(\partial \Omega)$ are compact, the subsequences $\left\{\widehat{u}_{m_{k}}\right\},\left\{\widehat{v}_{m_{k}}\right\}$ converge strongly respective to $\widehat{u}, \widehat{v}$ in $L^{2}(\Omega)$ and $L^{2}(\partial \Omega)$. 
From (2.5) dividing by $\left\|w_{m_{k}}\right\|_{E}^{2}$ we deduce that

$$
\begin{aligned}
& \limsup _{k \rightarrow+\infty}\left\{\frac{1}{2} \int_{\Omega}\left(h_{1}(x)\left|\nabla \widehat{u}_{m_{k}}\right|^{2}+a_{1}(x)\left|\widehat{u}_{m_{k}}\right|^{2}\right) d x-\frac{\lambda_{11}}{2} \int_{\Omega} \theta_{1}(x)\left|\widehat{u}_{m_{k}}\right|^{2} d x\right. \\
& +\frac{1}{2} \int_{\Omega}\left(h_{2}(x)\left|\nabla \widehat{v}_{m_{k}}\right|^{2}+a_{2}(x)\left|\widehat{v}_{m_{k}}\right|^{2}\right) d x-\frac{\lambda_{21}}{2} \int_{\Omega} \theta_{2}(x)\left|\widehat{v}_{m_{k}}\right|^{2} d x \\
& \left.-\int_{\Omega} \frac{H\left(x, w_{m_{k}}\right)}{\| w_{m_{k}}||_{E}^{2}} d x+\int_{\Omega} \frac{k_{1}(x) \widehat{u}_{m_{k}}+k_{2}(x) \widehat{v}_{m_{k}}}{\| w_{m_{k}}|| E} d x-\int_{\partial \Omega} h(x) \frac{R\left(x, w_{m_{k}}\right)}{\left\|w_{m_{k}}\right\|_{E}^{2}} d s\right\} \\
& \leq 0 .
\end{aligned}
$$

By $\left(\mathrm{H}_{1}\right)$-(ii) and (1.11) we have:

$$
\left|H\left(x, w_{m_{k}}\right)\right| \leq 2 \tau_{1}(x)\left(\left|u_{m_{k}}\right|+\left|v_{m_{k}}\right|\right), \tau_{1}(x) \in L^{2}(\Omega) .
$$

Hence

$$
\left|\int_{\Omega} \frac{H\left(x, w_{m_{k}}\right)}{\left\|w_{m_{k}}\right\|_{E}^{2}} d x\right| \leq \frac{2}{\left\|w_{m_{k}}\right\|_{E}}\left\|\tau_{1}\right\|_{L^{2}(\Omega)}\left(\left\|\widehat{u}_{m_{k}}\right\|_{L^{2}(\Omega)}+\left\|\widehat{v}_{m_{k}}\right\|_{L^{2}(\Omega)}\right) .
$$

Remark that $\left\{\left(\widehat{u_{m k}}\right\},\left\{\widehat{v_{m k}}\right)\right\}$ converge strongly in $L^{2}(\Omega)$, hence they are bounded in $L^{2}(\Omega)$.

Letting $k \rightarrow+\infty$, since $\left\|w_{m_{k}}\right\|_{E} \rightarrow+\infty$, we obtain:

$$
\limsup _{k \rightarrow+\infty} \int_{\Omega} \frac{H\left(x, w_{m_{k}}\right)}{\left\|w_{m_{k}}\right\|_{E}^{2}} d x=0
$$

Similarly, we also obtain

$$
\begin{aligned}
& \limsup _{k \rightarrow+\infty} \int_{\Omega} \frac{k_{1}(x) \widehat{u}_{m_{k}}+k_{2}(x) \widehat{v}_{m_{k}}}{\left\|u_{m_{k}}\right\|_{E}} d x=0, \\
& \limsup _{k \rightarrow+\infty} \int_{\partial \Omega} h(x) \frac{R\left(x, w_{m_{k}}\right)}{\left\|w_{m_{k}}\right\|_{E}^{2}} d s=0 .
\end{aligned}
$$

Moreover, we have

$$
\begin{aligned}
& \lim _{k \rightarrow+\infty} \int_{\Omega} \theta_{1}(x)\left|\widehat{u}_{m_{k}}\right|^{2} d x=\int_{\Omega} \theta_{1}(x)|\widehat{u}|^{2} d x, \\
& \lim _{k \rightarrow+\infty} \int_{\Omega} \theta_{2}(x)\left|\widehat{v}_{m_{k}}\right|^{2} d x=\int_{\Omega} \theta_{2}(x)|\widehat{v}|^{2} d x .
\end{aligned}
$$

Then from (2.6) and (2.8), (2.9), (2.10), we deduce that

$$
\begin{aligned}
& \limsup _{k \rightarrow+\infty}\left\{\int_{\Omega}\left(h_{1}(x)\left|\nabla \widehat{u}_{m_{k}}\right|^{2}+a_{1}(x)\left|\widehat{u}_{m_{k}}\right|^{2}\right) d x\right. \\
& \left.+\int_{\Omega}\left(h_{2}(x)\left|\nabla \widehat{v}_{m_{k}}\right|^{2}+a_{2}(x)\left|\widehat{v}_{m_{k}}\right|^{2}\right) d x\right\} \\
& \leq \lambda_{11} \int_{\Omega} \theta_{1}(x)|\widehat{u}|^{2} d x+\lambda_{21} \int_{\Omega} \theta_{2}(x)|\widehat{v}|^{2} d x .
\end{aligned}
$$


By Remark 1.1 and the variational characterization of $\lambda_{11}, \lambda_{21}$, we get:

$$
\begin{aligned}
& \lambda_{11} \int_{\Omega} \theta_{1}(x)|\widehat{u}|^{2} d x+\lambda_{21} \int_{\Omega} \theta_{2}(x)|\widehat{v}|^{2} d x \\
\leq & \int_{\Omega}\left(h_{1}(x)|\nabla \widehat{u}|^{2}+a_{1}(x)|\widehat{u}|^{2}\right) d x+\int_{\Omega}\left(h_{2}(x)|\nabla \widehat{v}|^{2}+a_{2}(x)|\widehat{v}|^{2}\right) d x \\
\leq & \liminf _{k \rightarrow+\infty}\left\{\int_{\Omega}\left(h_{1}(x)\left|\nabla \widehat{u}_{m_{k}}\right|^{2}+a_{1}(x)\left|\widehat{u}_{m_{k}}\right|^{2}\right) d x\right. \\
& \left.+\int_{\Omega}\left(h_{2}(x)\left|\nabla \widehat{v}_{m_{k}}\right|^{2}+a_{2}(x)\left|\widehat{v}_{m_{k}}\right|^{2}\right) d x\right\} \\
\leq & \limsup _{k \rightarrow+\infty}\left\{\int_{\Omega}\left(h_{1}(x)\left|\nabla \widehat{u}_{m_{k}}\right|^{2}+a_{1}(x)\left|\widehat{u}_{m_{k}}\right|^{2}\right) d x\right. \\
& \left.+\int_{\Omega}\left(h_{2}(x)\left|\nabla \widehat{v}_{m_{k}}\right|^{2}+a_{2}(x)\left|\widehat{v}_{m_{k}}\right|^{2}\right) d x\right\} \\
\leq & \lambda_{11} \int_{\Omega} \theta_{1}(x)|\widehat{u}|^{2} d x+\lambda_{21} \int_{\Omega} \theta_{2}(x)|\widehat{v}|^{2} d x .
\end{aligned}
$$

Thus, these inequalities are indeed equalities and we have

$$
\begin{aligned}
& \lim _{k \rightarrow+\infty}\left\{\int_{\Omega}\left(h_{1}(x)\left|\nabla \widehat{u}_{m_{k}}\right|^{2}+a_{1}(x)\left|\widehat{u}_{m_{k}}\right|^{2}\right) d x\right. \\
& \left.+\int_{\Omega}\left(h_{2}(x)\left|\nabla \widehat{v}_{m_{k}}\right|^{2}+a_{2}(x)\left|\widehat{v}_{m_{k}}\right|^{2}\right) d x\right\} \\
= & \int_{\Omega}\left(h_{1}(x)|\nabla \widehat{u}|^{2}+a_{1}(x)|\widehat{u}|^{2}\right) d x+\int_{\Omega}\left(h_{2}(x)|\nabla \widehat{v}|^{2}+a_{2}(x)|\widehat{v}|^{2}\right) d x \\
= & \lambda_{11} \int_{\Omega} \theta_{1}(x)|\widehat{u}|^{2} d x+\lambda_{21} \int_{\Omega} \theta_{2}(x)|\widehat{v}|^{2} d x .
\end{aligned}
$$

On the other hand from (2.11) and remark that $\left\|\widehat{w}_{m_{k}}\right\|_{E}=1$ we infer that:

$$
\lim _{k \rightarrow+\infty}\left\|\widehat{w}_{m_{k}}\right\|_{E}=\|\widehat{w}\|_{E}=1 \text {. }
$$

Hence $\widehat{w}=(\widehat{u}, \widehat{v}) \neq 0$.

By again the variational characterization of $\lambda_{11}, \lambda_{21}$ we deduce that

$$
\begin{aligned}
\int_{\Omega}\left(h_{1}(x)|\nabla \widehat{u}|^{2}+a_{1}(x)|\widehat{u}|^{2}\right) d x & =\lambda_{11} \int_{\Omega} \theta_{1}(x)|\widehat{u}|^{2} d x, \\
\int_{\Omega}\left(h_{2}(x)|\nabla \widehat{v}|^{2}+a_{2}(x)|\widehat{v}|^{2}\right) d x & =\lambda_{21} \int_{\Omega} \theta_{2}(x)|\widehat{v}|^{2} d x .
\end{aligned}
$$

This implies from definition of eigenfunctions $\varphi_{11}(x)$ and $\varphi_{21}(x)$ that:

- If $\widehat{u} \neq 0, \widehat{v} \neq 0$, then $\widehat{u}(x)= \pm \varphi_{11}(x), \widehat{v}(x)= \pm \varphi_{21}(x)$.

- If $\widehat{u} \neq 0, \widehat{v}=0$, then $\widehat{u}(x)= \pm \varphi_{11}(x)$.

- If $\widehat{u}=0, \widehat{v} \neq 0$, then $\widehat{v}(x)= \pm \varphi_{21}(x)$.

Next, we will consider the following cases:

Let $\widehat{u}_{m_{k}} \rightarrow \widehat{u}=\varphi_{11}, \widehat{v}_{m_{k}} \rightarrow \widehat{v}=\varphi_{21}$ as $k \rightarrow+\infty$ in $L^{2}(\Omega)$ and $L^{2}(\partial \Omega)$. 
Firstly by the variational characterization of $\lambda_{11}$ and $\lambda_{21}$ we have

$$
\begin{aligned}
& \int_{\Omega}\left(h_{1}(x)\left|\nabla u_{m_{k}}\right|^{2}+a_{1}(x)\left|u_{m_{k}}\right|^{2}\right) d x+\int_{\Omega}\left(h_{2}(x)\left|\nabla v_{m_{k}}\right|^{2}+a_{2}(x)\left|v_{m_{k}}\right|^{2}\right) d x \\
\geq & \lambda_{11} \int_{\Omega} \theta_{1}(x)\left|u_{m_{k}}\right|^{2} d x+\lambda_{21} \int_{\Omega} \theta_{2}(x)\left|v_{m_{k}}\right|^{2} d x, m=1,2, \ldots
\end{aligned}
$$

Hence from (2.5) one get

$$
\begin{aligned}
& \quad-\int_{\Omega} H\left(x, w_{m_{k}}\right) d x-\int_{\partial \Omega} h(x) R\left(x, w_{m_{k}}\right) d s \\
& \quad+\int_{\Omega}\left[k_{1}(x) u_{m_{k}}(x)+k_{2}(x) v_{m_{k}}(x)\right] d x \\
& \leq I\left(w_{m_{k}}\right) \leq c, k=1,2, \ldots
\end{aligned}
$$

After dividing (2.12) by $\left\|w_{m_{k}}\right\|_{E}$, letting $\lim \sup _{k \rightarrow+\infty}$ and remark that $\lim _{k \rightarrow+\infty} \int_{\Omega}\left[k_{1}(x) \widehat{u}_{m_{k}}(x)+k_{2}(x) \widehat{v}_{m_{k}}(x)\right] d x=\int_{\Omega}\left[k_{1}(x) \varphi_{11}(x)+k_{2}(x) \varphi_{21}(x)\right] d x$.

We get

$$
\begin{aligned}
& \limsup _{k \rightarrow+\infty}\left\{\int_{\Omega} \frac{H\left(x, w_{m_{k}}\right)}{\left\|w_{m_{k}}\right\|_{E}} d x+\int_{\partial \Omega} h(x) \frac{R\left(x, w_{m_{k}}\right)}{\left\|w_{m_{k}}\right\|_{E}} d s\right\} \\
\geq & \int_{\Omega}\left[k_{1}(x) \varphi_{11}(x)+k_{2}(x) \varphi_{21}(x)\right] d x .
\end{aligned}
$$

Lemma 2.1. Assume that $\widehat{u}_{m_{k}} \rightarrow \varphi_{11}, \widehat{v}_{m_{k}} \rightarrow \varphi_{21}$ in $L^{2}(\Omega)$ and $L^{2}(\partial \Omega)$ as $k \rightarrow+\infty$. Then:

$$
\text { i) } \limsup _{k \rightarrow+\infty} \int_{\Omega} \frac{H\left(x, w_{m_{k}}\right)}{\left\|w_{m_{k}}\right\|_{E}} d x=\frac{1}{2} \int_{\Omega}\left[F_{11}(x) \varphi_{11}+G_{11} \varphi_{21}\right] d x
$$

ii) $\limsup _{k \rightarrow+\infty} \int_{\partial \Omega} h(x) \frac{R\left(x, w_{m_{k}}\right)}{\left\|w_{m_{k}}\right\|_{E}} d s=\frac{1}{2} \int_{\partial \Omega} h(x)\left[P_{11}(x) \varphi_{11}+Q_{11} \varphi_{21}\right] d s$.

Proof. By (1.11) we have

$2 H\left(x, w_{m_{k}}\right)=\int_{0}^{u_{m_{k}}}\left[f\left(x, s, v_{m_{k}}\right)+f(x, s, 0)\right] d s+\int_{0}^{v_{m_{k}}}\left[g\left(x, u_{m_{k}}, t\right)+g(x, 0, t)\right] d t$.

Set $l_{k}=\left\|w_{m_{k}}\right\|_{E} \rightarrow+\infty$ as $k \rightarrow+\infty$.

Observe that by hypotheses $\left(\mathrm{H}_{1}\right)$ on $f(x, w), g(x, w)$ we have

$$
\begin{aligned}
& \left|\int_{0}^{u_{m_{k}}} f\left(x, s, v_{m_{k}}\right) d s-\int_{0}^{l_{k} \varphi_{11}} f\left(x, s, l_{k} \varphi_{21}\right) d s\right| \\
\leq & \left|\int_{0}^{u_{m_{k}}}\left[f\left(x, s, v_{m_{k}}\right)-f\left(x, s, l_{k} \varphi_{21}\right)\right] d s\right|+\left|\int_{l_{k} \varphi_{11}}^{\left.u_{m_{k}}\right)} f\left(x, s, l_{k} \varphi_{21}\right) d s\right| \\
\leq & \left|\int_{0}^{u_{m_{k}}} \frac{\partial f}{\partial t}\left(x, s, l_{k} \varphi_{21}+\delta\left(v_{m_{k}}-l_{k} \varphi_{21}\right)\right) \cdot\left(v_{m_{k}}-l_{k} \varphi_{21}\right) d s\right|
\end{aligned}
$$




$$
\begin{aligned}
& +\tau_{1}(x)\left|u_{m_{k}}-l_{k} \varphi_{11}\right| \\
\leq & \left|\int_{0}^{u_{m_{k}}} \frac{\partial g}{\partial s}\left(x, s, l_{k} \varphi_{21}+\delta\left(v_{m_{k}}-l_{k} \varphi_{21}\right)\right) d s \cdot\left(v_{m_{k}}-l_{k} \varphi_{21}\right)\right| \\
& +\tau_{1}(x)\left|u_{m_{k}}-l_{k} \varphi_{11}\right| \\
\leq & 2 \tau_{1}(x)\left|v_{m_{k}}-l_{k} \varphi_{21}\right|+\tau_{1}(x)\left|u_{m_{k}}-l_{k} \varphi_{11}\right|, \delta \in(0,1) .
\end{aligned}
$$

From this and remark that $\widehat{u}_{m_{k}}=\frac{u_{m_{k}}}{l_{k}}, \widehat{v}_{m_{k}}=\frac{v_{m_{k}}}{l_{k}}$, we get:

$$
\begin{aligned}
& \left|\frac{1}{l_{k}} \int_{0}^{u_{m_{k}}} f\left(x, s, v_{m_{k}}\right) d s-\frac{1}{l_{k}} \int_{0}^{l_{k} \varphi_{11}} f\left(x, s, l_{k} \varphi_{21}\right) d s\right| \\
\leq & 2 \tau_{1}(x)\left|\widehat{v}_{m_{k}}-\varphi_{21}\right|+\tau_{1}(x)\left|\widehat{u}_{m_{k}}-\varphi_{11}\right| .
\end{aligned}
$$

Similarly,

$$
\begin{aligned}
& \left|\frac{1}{l_{k}} \int_{0}^{u_{m_{k}}} f(x, s, 0) d s-\frac{1}{l_{k}} \int_{0}^{l_{k} \varphi_{11}} f(x, s, 0) d s\right| \\
\leq & \tau_{1}(x)\left|\widehat{u}_{m_{k}}-\varphi_{11}\right| .
\end{aligned}
$$

Combining (2.17), (2.18) we infer that

$$
\begin{aligned}
& \mid \int_{\Omega}\left\{\frac{1}{l_{k}} \int_{0}^{u_{m_{k}}}\left[f\left(x, s, v_{m_{k}}\right)+f(x, s, 0)\right] d s\right. \\
& \left.-\frac{1}{l_{k}} \int_{0}^{l_{k} \varphi_{11}}\left[f\left(x, s, l_{k} \varphi_{21}\right)+f(x, s, 0)\right] d s\right\} d x \mid \\
\leq & \int_{\Omega}\left\{2 \tau_{1}(x)\left|\left(\widehat{v}_{m_{k}}-\varphi_{21}\right)\right|+2 \tau_{1}(x)\left|\widehat{u}_{m_{k}}-\varphi_{11}\right|\right\} d x \\
\leq & 2\left\|\tau_{1}(x)\right\|_{L^{2}(\Omega)} \cdot\left\|\widehat{v}_{m_{k}}-\varphi_{21}\right\|_{L^{2}(\Omega)}+2\left\|\tau_{1}(x)\right\|_{L^{2}(\Omega)} \cdot\left\|\widehat{u}_{m_{k}}-\varphi_{11}\right\|_{L^{2}(\Omega)} .
\end{aligned}
$$

Letting $k \rightarrow+\infty$, since

$$
\lim _{k \rightarrow+\infty}\left\|\widehat{v}_{m_{k}}-\varphi_{21}\right\|_{L^{2}(\Omega)}=0, \lim _{k \rightarrow+\infty}\left\|\widehat{u}_{m_{k}}-\varphi_{11}\right\|_{L^{2}(\Omega)}=0
$$

we deduce that

$$
\begin{aligned}
& \limsup _{k \rightarrow+\infty} \int_{\Omega}\left\{\frac{1}{l_{k}} \int_{0}^{u_{m_{k}}}\left[f\left(x, s, v_{m_{k}}\right)+f(x, s, 0)\right] d s\right\} d x \\
= & \limsup _{k \rightarrow+\infty} \int_{\Omega}\left\{\frac{1}{l_{k}} \int_{0}^{l_{k} \varphi_{11}}\left[f\left(x, s, l_{k} \varphi_{21}\right)+f(x, s, 0)\right] d s\right\} d x .
\end{aligned}
$$

Set $s=y \varphi_{11}(x), d s=\varphi_{11}(x) d y$, we get

$$
\begin{aligned}
& \int_{0}^{l_{k} \varphi_{11}}\left[f\left(x, s, l_{k} \varphi_{21}\right)+f(x, s, 0)\right] d s \\
= & \int_{0}^{l_{k}}\left[f\left(x, y \varphi_{11}, l_{k} \varphi_{21}\right)+f\left(x, y \varphi_{11}, 0\right)\right] \varphi_{11} d y .
\end{aligned}
$$


Remark that $l_{k}=\left\|w_{m_{k}}\right\|_{E} \rightarrow+\infty$ as $k \rightarrow+\infty$, hence

$$
\begin{aligned}
& \limsup _{k \rightarrow+\infty} \int_{\Omega}\left\{\frac{1}{l_{k}} \int_{0}^{u_{m_{k}}}\left[f\left(x, s, v_{m_{k}}\right)+f(x, s, 0)\right] d s\right\} d x \\
= & \limsup _{k \rightarrow+\infty} \int_{\Omega}\left\{\frac{1}{l_{k}} \int_{0}^{l_{k}}\left[f\left(x, y \varphi_{11}, l_{k} \varphi_{21}\right)+f\left(x, y \varphi_{11}, 0\right)\right] d y\right\} \varphi_{11} d x \\
= & \int_{\Omega} F_{11}(x) \varphi_{11}(x) d x .
\end{aligned}
$$

Similarly, we also derive that

$$
\limsup _{k \rightarrow+\infty} \int_{\Omega}\left\{\frac{1}{l_{k}} \int_{0}^{v_{m_{k}}}\left[g\left(x, u_{m_{k}}, t\right)+g(x, 0, t)\right] d s\right\} d x=\int_{\Omega} G_{11}(x) \varphi_{21}(x) d x,
$$

where $F_{11}(x), G_{11}(x)$ are given in (1.13a).

Combining (2.19), (2.20) we obtain:

$$
\limsup _{k \rightarrow+\infty} \int_{\Omega} 2 \frac{H\left(x, w_{m_{k}}\right)}{\left\|w_{m_{k}}\right\|_{E}} d x=\int_{\Omega}\left[F_{11}(x) \varphi_{11}(x)+G_{11}(x) \varphi_{21}(x)\right] d x .
$$

By computations as those above we also have

$\lim _{k \rightarrow+\infty} \sup \int_{\partial \Omega} 2 h(x) \frac{R\left(x, w_{m_{k}}\right)}{\left\|w_{m_{k}}\right\|_{E}} d x=\int_{\partial \Omega} h(x)\left[P_{11}(x) \varphi_{11}(x)+Q_{11}(x) \varphi_{21}(x)\right] d s$, where $P_{11}(x), Q_{11}(x)$ are given in (1.13a).

Lemma 2.1 is proved.

By Lemma 2.1, from (2.13) and using (2.14), (2.15), we deduce that:

$$
\begin{aligned}
& \int_{\Omega}\left[F_{11}(x) \varphi_{11}(x)+G_{11}(x) \varphi_{21}(x)\right] d x \\
& +\int_{\partial \Omega} h(x)\left[P_{11}(x) \varphi_{11}(x)+Q_{11}(x) \varphi_{21}(x)\right] d s \\
\geq & 2 \int_{\Omega}\left[k_{1}(x) \varphi_{11}(x)+k_{2}(x) \varphi_{21}(x)\right] d x .
\end{aligned}
$$

That is

$$
L_{11} \geq 2 \int_{\Omega}\left[k_{1}(x) \varphi_{11}+k_{2}(x) \varphi_{21}\right] d x
$$

which contradicts (1.15a).

Now, assume that $\widehat{u}_{m_{k}} \rightarrow \widehat{u}=\varphi_{11}, \widehat{v}_{m_{k}} \rightarrow \widehat{v}=-\varphi_{21}$ as $k \rightarrow+\infty$ in $L^{2}(\Omega)$ and $L^{2}(\partial \Omega)$.

Remark that with the similar computations as those used in the proof of the Lemma 2.1, we get

$$
\limsup _{k \rightarrow+\infty} \int_{\Omega} \frac{H\left(x, w_{m_{k}}\right)}{\left\|w_{m_{k}}\right\|_{E}} d x=\frac{1}{2} \int_{\Omega}\left[F_{12}(x) \varphi_{11}(x)-G_{12}(x) \varphi_{21}(x)\right] d x,
$$


$\limsup _{k \rightarrow+\infty} \int_{\partial \Omega} h(x) \frac{R\left(x, w_{m_{k}}\right)}{\left\|w_{m_{k}}\right\|_{E}} d s=\frac{1}{2} \int_{\partial \Omega} h(x)\left[P_{12}(x) \varphi_{11}(x)-Q_{12}(x) \varphi_{21}(x)\right] d s$.

After dividing (2.12) by $\left\|w_{m_{k}}\right\|_{E}$, letting $\lim \sup _{k \rightarrow+\infty}$ and remark that

$\lim _{k \rightarrow+\infty} \int_{\Omega}\left[k_{1}(x) \widehat{u}_{m_{k}}(x)+k_{2}(x) \widehat{v}_{m_{k}}(x)\right] d x=\int_{\Omega}\left[k_{1}(x) \varphi_{11}(x)-k_{2}(x) \varphi_{21}(x)\right] d x$.

We obtain:

$$
\begin{aligned}
& \int_{\Omega}\left[F_{12}(x) \varphi_{11}(x)-G_{12}(x) \varphi_{21}(x)\right] d x \\
+ & \int_{\partial \Omega} h(x)\left[P_{12}(x) \varphi_{11}(x)-Q_{12}(x) \varphi_{21}(x)\right] d s \\
\geq & 2 \int_{\Omega}\left[k_{1}(x) \varphi_{11}(x)-k_{2}(x) \varphi_{21}(x)\right] d x .
\end{aligned}
$$

This implies that

$$
L_{12} \geq 2 \int_{\Omega}\left[k_{1}(x) \varphi_{11}-k_{2}(x) \varphi_{21}\right] d x
$$

which contradicts $(1.16 \mathrm{a})$.

Similarly, in the cases when $\widehat{u}_{m_{k}} \rightarrow \widehat{u}=-\varphi_{11}, \widehat{v}_{m_{k}} \rightarrow \widehat{v}=-\varphi_{21}$ and when $\widehat{u}_{m_{k}} \rightarrow \widehat{u}=-\varphi_{11}, \widehat{v}_{m_{k}} \rightarrow \widehat{v}=\varphi_{21}$ we obtain the following respective inequalities

and

$$
L_{22} \leq 2 \int_{\Omega}\left[k_{1}(x) \varphi_{11}+k_{2}(x) \varphi_{21}\right] d x
$$

$$
L_{21} \geq 2 \int_{\Omega}\left[k_{1}(x) \varphi_{11}-k_{2}(x) \varphi_{21}\right] d x
$$

which contradict (1.15a) and (1.16a).

Now, we consider the case when $\widehat{u}_{m_{k}} \rightarrow \widehat{u}=\varphi_{11}(x)$ and $\widehat{v}_{m_{k}} \rightarrow \widehat{v}=0$ as $k \rightarrow+\infty$ in $L^{2}(\Omega)$ and $L^{2}(\partial \Omega)$.

By similar computations as those used in the proof of Lemma 2.1, we obtain

$$
\begin{aligned}
& \limsup _{k \rightarrow+\infty} \int_{\Omega}\left\{\frac{1}{l_{k}} \int_{0}^{u_{m_{k}}}\left[f\left(x, s, v_{m_{k}}\right)+f(x, s, 0)\right] d s\right\} d x \\
= & \limsup _{k \rightarrow+\infty} \int_{\Omega}\left\{\frac{1}{l_{k}} \int_{0}^{l_{k}} 2 f\left(x, y \varphi_{11}, 0\right) d y\right\} \varphi_{11}(x) d x
\end{aligned}
$$

and

$$
\limsup _{k \rightarrow+\infty} \int_{\Omega}\left\{\frac{1}{l_{k}} \int_{0}^{v_{m_{k}}}\left[g\left(x, u_{m_{k}}, t\right)+g(x, 0, t)\right] d t\right\} d x=0,
$$

where $l_{k}=\left\|w_{m_{k}}\right\|_{E} \rightarrow+\infty$ as $k \rightarrow+\infty$.

From this and remark (1.11) we arrive at

$$
\limsup _{k \rightarrow+\infty} \int_{\Omega} \frac{H\left(x, w_{m_{k}}\right)}{\left\|w_{m_{k}}\right\|_{E}} d x=\limsup _{k \rightarrow+\infty} \int_{\Omega}\left\{\frac{1}{l_{k}} \int_{0}^{l_{k}} f\left(x, y \varphi_{11}, 0\right) d y\right\} \varphi_{11}(x) d x
$$




$$
=\int_{\Omega} F_{10}(x) \varphi_{11}(x) d x
$$

where $F_{10}(x)$ is given by $(1.13 \mathrm{~b})$.

Similarly

$$
\limsup _{k \rightarrow+\infty} \int_{\partial \Omega} h(x) \frac{R\left(x, w_{m_{k}}\right)}{\left\|w_{m_{k}}\right\|_{E}} d s=\int_{\partial \Omega} h(x) P_{10}(x) \varphi_{11}(x) d s,
$$

where $P_{10}(x)$ is given by $(1.13 \mathrm{~b})$.

From (2.13) we get

$$
\int_{\Omega} F_{10}(x) \varphi_{11}(x) d x+\int_{\partial \Omega} h(x) P_{10}(x) \varphi_{11}(x) d s \geq \int_{\Omega} k_{1}(x) \varphi_{11}(x) d x,
$$

which gives

$$
\int_{\Omega} k_{1}(x) \varphi_{11}(x) d x \leq L_{10}
$$

We get a contradiction with (1.15b).

By same arguments, if $\widehat{u}_{m_{k}} \rightarrow \widehat{u}=-\varphi_{11}(x)$ and $\widehat{v}_{m_{k}} \rightarrow \widehat{v}=0$ as $k \rightarrow+\infty$ in $L^{2}(\Omega)$ and $L^{2}(\partial \Omega)$. From $(2.12)$ we obtain:

$$
-\int_{\Omega} F_{20}(x) \varphi_{11}(x) d x-\int_{\partial \Omega} h(x) P_{20}(x) \varphi_{21}(x) d s \geq-\int_{\Omega} k_{1}(x) \varphi_{11}(x) d x .
$$

This implies

$$
L_{20} \leq \int_{\Omega} k_{1}(x) \varphi_{11}(x) d x
$$

which contradicts (1.15b).

In the cases when $\widehat{u}_{m_{k}} \rightarrow \widehat{u}=0$ and $\widehat{v}_{m_{k}} \rightarrow \widehat{v}= \pm \varphi_{21}(x)$ as $k \rightarrow+\infty$ in $L^{2}(\Omega)$ and $L^{2}(\partial \Omega)$, by similar computations used above we arrive at contradictions with (1.16b) as follows:

$$
\int_{\Omega} k_{2}(x) \varphi_{21}(x) d x \leq L_{01}
$$

and

$$
\int_{\Omega} k_{2}(x) \varphi_{21}(x) d x \geq L_{02} .
$$

Thus the functional $I$ given by (2.1) is coercive on $E$ and Proposition 2.2 is proved.

Proof of Theorem 1.1. By Proposition 2.2 and the weak lower semicontinuity of the functional $I$ (see Remark 2.1), applying the Minimum Principle (see [12, p. 4, Theorem 1.2]), the functional $I$ has a global minimum and by $\left(\mathrm{H}_{1}\right)$ the problem (1.1) admits a nontrivial weak solution in $E$. 
Remark 2.3. Since $\|\widehat{w}\|_{E}^{2}=\|\widehat{u}\|_{E_{1}}^{2}+\|\widehat{v}\|_{E_{2}}^{2}$, we would also consider more general cases such that $\widehat{u}=a \varphi_{11}, \widehat{v}=b \varphi_{21}$, where $a^{2}+b^{2}=1$. So the hypotheses (H2) would be changed by following condition more general

$$
\begin{aligned}
& \int_{\Omega}\left(a F(x) \varphi_{11}(x)+b G(x) \varphi_{21}(x)\right) d x \\
& +\int_{\partial \Omega} h(x)\left[a P(x) \varphi_{11}(x)+b Q(x) \varphi_{21}(x)\right] d x \\
< & 2 \int_{\Omega}\left(a k_{1}(x) \varphi_{11}(x)+b k_{2}(x) \varphi_{21}(x)\right) d x, \forall a, b \in R: a^{2}+b^{2}=1,
\end{aligned}
$$

where

$$
\begin{aligned}
& F(x)=\lim _{\tau \rightarrow+\infty} \frac{1}{\tau} \int_{0}^{\tau}\left[f\left(x, y a \varphi_{11}, \tau b \varphi_{21}\right)+f\left(x, y a \varphi_{11}, 0\right)\right] d y \\
& G(x)=\lim _{\tau \rightarrow+\infty} \frac{1}{\tau} \int_{0}^{\tau}\left[g\left(x, \tau a \varphi_{11}, y b \varphi_{21}\right)+g\left(x, 0, y b \varphi_{21}\right)\right] d y \\
& P(x)=\lim _{\tau \rightarrow+\infty} \frac{1}{\tau} \int_{0}^{\tau}\left[p\left(x, y a \varphi_{11}, \tau b \varphi_{21}\right)+p\left(x, y a \varphi_{11}, 0\right)\right] d y \\
& Q(x)=\lim _{\tau \rightarrow+\infty} \frac{1}{\tau} \int_{0}^{\tau}\left[q\left(x, \tau a \varphi_{11}, y b \varphi_{21}\right)+q\left(x, 0, y b \varphi_{21}\right)\right] d y
\end{aligned}
$$

and the proof of Proposition 2.2 is more simple. However, this condition is more strict and difficult to check.

Remark 2.4. Remark if we replace the inequalities in the hypotheses $\left(\mathrm{H}_{2}\right)-(\mathrm{i})$, (ii) by following inverse inequalities:

$$
\text { (i) } \begin{aligned}
L_{11} & >2 \int_{\Omega}\left[k_{1}(x) \varphi_{11}(x)+k_{2}(x) \varphi_{21}(x)\right] d x>L_{22}, \\
L_{12}>2 \int_{\Omega}\left[k_{1}(x) \varphi_{11}(x)-k_{2}(x) \varphi_{21}(x)\right] d x>L_{21} . & \\
L_{10} & >\int_{\Omega} k_{1}(x) \varphi_{11}(x) d x>L_{20}, \\
L_{01} & >\int_{\Omega} k_{2}(x) \varphi_{21}(x) d x>L_{02},
\end{aligned}
$$

then by applying Saddle Point Theorem, we can prove that the problem (1.1), (1.2) also has at least weak solution in $E$.

Acknowledgement. The authors would like to thank the referees for their suggestions and helpful comments which improved the presentation of the paper.

\section{References}

[1] A. Anane and J. P Gossez, Strongly nonlinear elliptic problems near resonance a variational approach, Comm. Partial Differential Equation 15 (1990), no. 8, 1141-1159. 
[2] D. Arcoya and L. Orsina, Landesman-Lazer condition and quasilinear elliptic equations, Nonlinear Anal. 28 (1997), no. 10, 1623-1632.

[3] L. Boccando, P. Drábek, and M. Kučera, Landesman-Lazer conditions for strongly nonlinear boundary value problems, Comment. Math. Univ. Carolin. 30 (1989), no. 3, 411427.

[4] N. T. Chung and H. Q. Toan, Existence result for nonuniformly degenerate semilinear elliptic systems in $\mathbb{R}^{N}$, Glasgow Math. J. 51 (2009), 561-570.

[5] D. Gilbarg and N. Trudinger, Elliptic Partial Differential Equations of Second Order, Springer Verlag Berlin, 2001.

[6] T. T. M. Hang and H. Q. Toan, On existence of weak solutions of Neumann problem for quasilinear elliptic equations involving p-Laplacian in an unbounded domain, Bull. Korean Math. Soc 48 (2011), no. 6, 1169-1182.

[7] D. A. Kandilakis and M. Magiropoulos, A p-Laplacian system with resonance and nonlinear boundary conditions on an unbounded domain, Comment. Math. Univ. Carolin. 48 (2007), no. 1, 59-68.

[8] N. Lam and G. Lu, Existence and multiplicity of solutions to equations of N-Laplacian type with critical exponential growth in $\mathbb{R}^{N}$, J. Funct. Anal. 262 (2012), no. 3, 11321165.

[9] M. Lucia, P. Magrone, and Huan-Songzhou, A Dirichlet problem with asymptotically linear and changing sign nonlinearity, Rev. Mat. Complut. 16 (2003), no. 2, 465-481.

[10] Q. A. Ngo and H. Q. Toan, Existence of solutions for a resonant problem under Landesman-Lazer condition, Electron. J. Differential Equations 2008 (2008), no. 98, $1-10$.

[11] Some remarks on a class of nonuniformly elliptic equations of p-Laplacian type, Acta Appl. Math. 106 (2009), no. 2, 229-239.

[12] M. Struwe, Variational Methods, Second edition, Springer Verlag, 2008.

[13] H. Q. Toan and N. T. Chung, Existence of weak solutions for a class of nonuniformly nonlinear elliptic equations in unbounded domains, Nonlinear Anal. 70 (2009), no. 11, 3987-3996.

[14] P. Tomiczek, A generalization of the Landesman-Lazer condition, Electron. J. Differential Equations 2001 (2001), no. 4, 1-11.

[15] Z.-Q. Ou and C.-L. Tang, Resonance problems for the p-Laplacian systems, J. Math. Anal. Appl. 345 (2008), no. 1, 511-521.

[16] N. B. Zographopoulos, p-Laplacian systems on resonance, Appl. Anal. 83 (2004), no. 5, 509-519.

HOANG QUOC TOAN

Department of Mathematics

HANOI UNIVERSITY OF SCIENCE

334 Nguyen Trai, Thanh Xuan, Hanoi, Vietnam

E-mail address: hq_toan@yahoo.com

Bui QuOC Hung

FACUlty of InFormation TeChNOLOGy

Le Quy Don Technical University

236 Hoang Quoc Viet, Cau Giay, Hanoi, Vietnam

E-mail address: quochung2806@yahoo.com 